\title{
Mystery of the brain metastatic disease in breast cancer patients: improved patient stratification, disease prediction and targeted prevention on the horizon?
}

\author{
Jiri Polivka Jr ${ }^{1,2} \cdot$ Milena Kralickova ${ }^{1,2}$ - Jiri Polivka ${ }^{3}$ - Christina Kaiser ${ }^{4,5,6}$. \\ Walther Kuhn ${ }^{4,5,6}$ • Olga Golubnitschaja ${ }^{5,6,7}$
}

Received: 12 January 2017 / Accepted: 14 February 2017 /Published online: 13 March 2017

(C) European Association for Predictive, Preventive and Personalised Medicine (EPMA) 2017

\begin{abstract}
The breast cancer (BC) diagnosis currently experiences the epidemic evolution with more than half of million deaths each year. Despite screening programmes applied and treatments available, breast cancer patients frequently develop distant metastases. The brain is one of the predominant sites of the metastatic spread recorded for more than $20 \%$ of BC patients, in contrast to the general population, where brain tumours are rarely diagnosed. Although highly clinically relevant, the brain tumour mystery in the cohort of breast cancer patients has not been yet adequately explained. This review summarises currently available information on the risk factors predicting brain metastases in $\mathrm{BC}$ patients to motivate the relevant scientific areas to explore the data/facts available and elucidate disease-specific mechanisms that are of a great clinical utility.
\end{abstract}

Olga Golubnitschaja

Olga.Golubnitschaja@ukb.uni-bonn.de

1 Department of Histology and Embryology, Faculty of Medicine in Plzen, Charles University, Plzen, Czech Republic

2 Biomedical Centre, Faculty of Medicine in Plzen, Charles University, Plzen, Czech Republic

3 Department of Neurology, Faculty Hospital Plzen, Plzen, Czech Republic

4 Department of Gynaecology, Medical Faculty, Friedrich-Wilhelms-University of Bonn, Bonn, Germany

5 Breast Cancer Research Centre, Friedrich-Wilhelms-University of Bonn, Bonn, Germany

6 Centre for Integrated Oncology Cologne-Bonn, Friedrich-Wilhelms-University of Bonn, Sigmund-Freud-Str 25, 53105 Bonn, Germany

7 Department of Radiology, Friedrich-Wilhelms-University of Bonn, Sigmund-Freud-Str 25, 53105 Bonn, Germany
Keywords Breast cancer · Brain metastasis · Risk factors · Multilevel diagnostics $\cdot$ Patient stratification $\cdot$ Predictive preventive and personalised medicine

\section{Introduction}

The breast cancer (BC) diagnosis currently experiences the epidemic evolution with more than half of million deaths each year [1]. This major public problem affects several million of patients and their families worldwide, healthcare systems and the society at large. Despite screening programmes applied and treatments available, $\mathrm{BC}$ patients frequently develop distant metastases during the course of their disease - some of them are particularly aggressive characterised by the rapid appearance and progression of the metastatic disease, poor prognosis and fatal outcomes in a very short period of time after the clinical onset of the original tumour in breast. Although predominant sites of the secondary tumours are generally known for $\mathrm{BC}$ patients, any valid patient stratification is still underdeveloped enabling to predict individual predisposition to the metastatic spread into targeted distant organs as well particularly aggressive character of the metastatic disease. Due to several good reasons introduced and discussed in detail below, specifically brain metastatic disease should receive a particular attention in $\mathrm{BC}$ patient cohorts.

On one side, primary brain tumour is a rare disorder responsible for high mortality and morbidity: relatively low prevalence in general population is ranging between 12.0 and 15.5 per 100,000 person-year for different countries and regions worldwide [2-5]. On the other side, the brain is one of the predominant sites of the metastatic spread recorded for more than $20 \%$ of breast cancer patients in several individual subgroups $[6,7]$. Although highly clinically relevant, this mystery has not been yet adequately explained. Which 
specifics of $\mathrm{BC}$ do predispose the affected individuals to brain tumours which are rather unusual in the general population? Which patient cohorts and $\mathrm{BC}$ subtypes are particularly predisposed to brain metastases? Are there any reliable tools on the horizon which would enable healthcare providers to predict and effectively prevent brain metastases in stratified $\mathrm{BC}$ patients? Certainly, much more research efforts are needed to adequately address these questions. On a good way to that, in this article, we summarise currently available information to motivate the relevant scientific areas to explore the data/ facts available and elucidate disease-specific mechanisms that are of great clinical utility.

\section{Brain metastasis risk factors associated with the patient}

\section{Age}

The more aggressive disease phenotype is often accompanied with an early progression into the brain parenchyma and is clearly associated with the younger age of patients diagnosed with primary BC. Recent study revealed the development of brain metastases (BM) in 164 (7.3\%) out of 2248 patients with BC over a median follow-up of 54.2 months [8]. The patients' age at the time of diagnosis was acknowledged as the significant risk factor for a development of metastases in the brain. The cumulative BM 5 -year-incidence for the patients aged 35 years or younger was 20.5 versus $7.8 \%$ for patients aged between 36 and 59 years, and $7.5 \%$ for patients older than 60 years. In consensus, the multivariate analysis demonstrated the age of $\mathrm{BC}$ patients by 35 years and younger as an independent predictor for the BMrisk $(\mathrm{HR}=2.09$, 95\% CI 1.15-3.81, $P=0.016$ ). Likewise, the age younger than 35 years was proposed as the predictive factor for BM risks in another study that investigated 9524 breast cancer patients $(P<0.01)$ [9]. The explorative analysis that used the database containing information on 10,782 $\mathrm{BC}$ patients has identified a clear association of younger age of $\mathrm{BC}$ patients with a significantly higher prevalence of BM development $(P<0.001)[10]$. Recorded cumulative incidence of $\mathrm{BM}$ was significantly higher for $\mathrm{BC}$ patients younger than 50 years $(20.8 \%)$ compared to the older ones $(9.7 \%)$ by observing follow-up during 5 years [11]. A series of independent studies which differed from each other by experimental design and patient subgroups involved, have, nevertheless, confirmed these main findings [12, 13], namely:

1. Younger BC patients, in general, are at significantly higher risk of the BM development.

2. BC patients younger than 35 years are at the extraordinarily high risk of the metastatic disease developed in the brain.

On a positive note, several studies demonstrated that amongst those $\mathrm{BC}$ patients who developed $\mathrm{BM}$, the younger age was associated with better prognosis and overall survival compared to the older patients with the same diagnosis $[8,14]$. Within the context of the below listed facts, this particular finding strongly substantiates the meaning of new screening programmes and timely treatments to be created specifically for the young subpopulations, in order to effectively optimise healthcare in the area of breast cancer.

\section{Menopausal status}

Menopausal status is the other factor which may be predictable for the increased risk of BM in patients with early detected BC. Hence, premenopausal women sustainably demonstrate higher cumulative risks for BM development during the 5 years $(3.8$ vs. $2.8 \%), 10$ years $(6.2$ vs. $4.2 \%)$, and 15 years (7.1 vs. $4.6 \%)$ of the follow-up observations performed after the initial $\mathrm{BC}$ has been diagnosed $(P<0.01)$ [9]. Indeed, more recent study performed with 2136 metastatic breast cancer patients has confirmed that in contrast to premenopausal women, the postmenopausal ones are at lower risk of $\mathrm{BM}$ development $(\mathrm{HR}=0.68,95 \%$ CI $0.54-0.86$, $P=0.001)$ [12]. The significance of this risk impacted by the menopausal status $(P=0.008)$ has been further confirmed by an independent study [10]. Thus, the premenopausal status at the time of $\mathrm{BC}$ diagnosis is considered as an additional risk factor for the brain metastatic lesions.

\section{Brain metastasis risk factors associated with specific characteristics of primary breast tumours}

\section{Histopathologically identifiable risk factors}

Several specific histopathological characteristics of primary breast tumours have been characterised as associated with an increased risk of BM development. The nodal status, tumour grade and the tumour size are strongly predictive for the $\mathrm{BM}$ occurrence in patients with primary BC [9]. The cumulative incidence of BM has been demonstrated to be higher for the patients having more than four positive lymph nodes $(8.5 \%$ at 10 years follow-up, $P<0.01)$, tumour grade $3(7.8 \%$ at 10 years follow-up, $P<0.01)$ and the tumour size more than $2 \mathrm{~cm}(6.8 \%$ at 10 years follow-up, $P<0.01)$. The higher BC tumour grade was identified as strongly associated with BM risk $(P=0.002)$ in an independent study [13]. Significantly more $\mathrm{BC}$ patients with $\mathrm{BM}$ than without $\mathrm{BM}$ had tumour of higher histological grade (grade $3,78.9$ vs. $30.2 \% ; P=0.001$ ) as confirmed by the recently performed issue-dedicated study [15]. The increased incidence of BM correlates well with greater likelihood of axillary lymph node metastases from the primary tumour $(P<0.001)$. Another study showed the nodal positivity $(\mathrm{HR}=2.4,95 \% \mathrm{CI} 1.1-5.1, P=0.028)$ and larger primary tumours $(\mathrm{HR}=1.9,95 \% \mathrm{CI} 1.2-3.6, P=0.02)$ 
to be two independent predictors of more frequent $\mathrm{BM}$ development in BC patients [16].

Further, the infiltrating ductal carcinoma (IDC) classified by WHO as no special type (NST) carcinoma increases BM risk compared to lower $\mathrm{BM}$ predisposition of the patients affected by the lobular carcinoma (ILC) as it has been demonstrated utilising the issue-dedicated multivariate analysis $(\mathrm{HR}=2.5,95 \% \mathrm{CI} 1.1-5.5, P=0.02)[10]$ that correlates well with the results demonstrated by an independent study (HR = 3.20 , 95\% CI 1.00-10.31) [11]. Thereby, the primary breast tumours bigger than $2 \mathrm{~cm}$ were associated with significantly higher BM risks in contrast to the tumours equal or smaller than $2 \mathrm{~cm}$ in their size (HR $=1.5,95 \%$ CI $1.1-2, P=0.01)$. Noteworthy, itself, the metastatic disease in $\mathrm{BC}$ recorded either by local or distant metastatic spread significantly increases the risk for the development of metastases in the brain: enhanced BM risks calculated for the lymph node metastases are $\mathrm{HR}=2.55,95 \%$ CI $1.20-5.42$ and for the lung $\mathrm{HR}=1.99$, $95 \%$ CI $1.04-3.81$.

\section{Specific breast cancer subtypes significantly predisposing patients to the brain metastases}

Extremely high heterogeneity amongst breast tumours is well recognised by the field-dedicated experts. Hence, Jaak Janssens proposes the following: Should we speak about breast "cancers" instead of "cancer"? [17]. Proposed molecular biological tumour characterisation allows for an assignment of BC patients into well-distinguishable subgroups [18]. Actually accepted classification of $\mathrm{BC}$ based on the subtypespecific molecular profiles generally distinguishes between luminal A, luminal B, HER2-enriched and basal-like BC [19]. The luminal A and B subtypes abundantly express oestrogen (ER) and/or progesterone (PR) receptors. The HER2-enriched subtype overexpresses specifically the human epidermal growth factor-2 (HER2). Tumours characterised by low expression of the above listed biomarkers are called "triple negative" (TN) generally overlapping with the basallike BC subgroup [19, 20].

The distinct $\mathrm{BC}$ subtypes do perform the metastatic spread into preferentially targeted organs. Whereas luminal subtype experiences specifically skeletal and lung metastases [21, 22], the HER2-enriched as well as TN (basal-like) subtypes that frequently spread the metastases into the brain. To this end, the frequency of BM amongst metastatic $\mathrm{BC}$ patients is recorded to be as high as 10-20\% in HER2-enriched BC tumours but $20-30 \%$ in TN tumours [21-23]. The study based on the data collected from altogether 3726 patients diagnosed with early stages of BC revealed significantly increased prevalence of $\mathrm{BM}$ in patients characterised by HER2-positive but ER/PRnegative status ( $\mathrm{HR}=5.3,95 \% \mathrm{CI} 3.0-9.2)$ as well as by basal-like tumours ( $\mathrm{HR}=3.6,95 \% \mathrm{CI} 2.1-6.4$ ) versus luminal A subtype [21]. The 15-year cumulative incidences of BM in
HER2-positive but ER/PR-negative and basal-like subtypes of BC were 14.3 and $10.9 \%$, respectively, compared to $2.2 \%$ of BM in luminal A subgroup $(P<0.001)$. Hormone receptors and HER 2 expression status served as the predictive biomarkerpanel for BM in a number of other independent studies. Hence, oestrogen receptor negativity and HER2 overexpression both correlated significantly $(P<0.01)$ with BM appearance as the first metastatic site in 9524 young women affected by BC. Moreover, the cumulative incidence of secondary tumours in the central nervous system at any time of diagnosis was significantly higher in patients with HER2-positive than HER2negative disease $(6.8$ vs. $3.5 \%$ at 10 years; $P<0.01)$ and amongst patients with ER-negative compared to ER-positive primary tumours ( 7.8 vs. $4.0 \%$ at 10 years; $P<0.01$ ) [9]. Another study with 2685 metastatic BC patients and multivariate analysis performed revealed the ER negativity as an independent predictor of the increased BM risk compared to ERpositive tumours (HR $=2.8,95 \%$ CI $2.1-3.7, P<0.001)[10]$; thereby, the $\mathrm{BM}$ recurrence were more common in patients with HER2-positive tumours $(P=0.04)$. BC patients that suffered from BM were more likely to have ER-negative $(P<0.001)$ and HER 2 overexpressing $(P=0.001)$ tumours as demonstrated by another independent study [13].

However, the highest risk of $\mathrm{BM}$ occurrence $(\mathrm{HR}=4.2$, 95\% CI 2.3-7.6, $P<0.0001)$ specifically in the patients with invasive triple-negative breast tumours has been demonstrated amongst 2441 investigated ones [16]. Furthermore, patients with TN tumours show the shortest median interval between primary diagnosis and BM development (22 months) as compared with HER-positive (30 months) and ER-positive/ HER2-negative tumours (63.5 months). In another study based on the patient data collected from metastatic $\mathrm{BC}$, the HER2 positivity was associated with an increased risk of BM compared to HER2 negativity in tumour tissue $(\mathrm{HR}=2.58$, 95\% CI 1.52-4.39, $P<0.001$ ) [11]. The cumulative incidence of BM at 5-year follow-up was $26.1 \%$ for patients with HER2positive compared to $9.5 \%$ with HER2-negative tumours. Patients with TN breast tumours demonstrated the substantially increased risk of BM compared to hormone receptor-positive/HER2-negative tumours in another study as well (HR = 5.5, 95\% CI 1.4-21.2, $P=0.013$ ) [24]. Median duration recorded from the time point of the BC operation till BM occurrence was the shortest for TN patients (11.3 months) followed by HER2-positive ones (20.3 months) and others (24.1 months). Patients with TN (HR $=4.42,95 \%$ CI 2.86 $6.85, P<0.001)$ as well as HER2-enriched tumours (HR = $2.53,95 \%$ CI $1.57-4.07, P<0.001)$ were at increased risk for BM occurrence compared to the luminal A tumours as demonstrated by more recent study [8]. Interestingly, amongst patients aged 35 or younger, the risk of BM development was independent from subtypes of the primary breast tumours $(P=0.507)$. Finally, the hormone receptor status is an important risk factor for BM development specifically in patients 
with HER2-positive ER and PR-negative BC treated with anti-HER2 monoclonal antibodies: these patients demonstrate highly increased incidence of BM after the treatment, e.g. with trastuzumab (HR $=3.41,95 \%$ CI 1.33-8.71, $P=0.01)$ [25].

\section{Molecular and genetic biomarkers other than hormone and HER2 receptors as the risk factor for the brain metastases}

There is a huge number of molecular and genetics biomarkers other than ER, PR and HER2 receptors that were extensively studied in association with BM development in patients with early diagnosed as well as metastatic BC [26-29]. The substantial progress in cancer multi-omics disciplines (such as genomics, proteomics and transcriptomics) in recent years allowed the examination of distinct molecular patterns of BC tumours predicting the higher probability of development of $\mathrm{BM}$ after the disease recurrence.

Recently performed retrospective multivariate analysis based on the data collected from $591 \mathrm{BC}$ patients demonstrated the association of the overexpressed cellular proliferation biomarker Ki67 with the risk of BM development $(\mathrm{HR}=3.9$, 95\% CI 1.2-12.9, $P=0.026$ ) [24]. Contextually, the study that analysed 198 metastatic BCs has demonstrated a significantly increased risk of $\mathrm{BM}$ occurrence in patients with the tumour Ki67 overexpression $(\mathrm{HR}=2.76,95 \%$ CI $1.70-4.48$, $P<0.001$ ) as well as cytoplasmic expression of Rad51 (HR $1.87,95 \%$ CI 1.14-3.08, $P=0.014$ ) [30]. To this end, however, it should be mentioned that the predictive power of Ki67 is not strong enough, if used as an individual biomarker; in contrast, a multilevel biomarker panel might be of more clinical utility.

Further, expression levels of the panel of 17 well-justified proteins and their potential associations with the first metastatic site were investigated in the study utilising the data of 2032 $\mathrm{BC}$ patients in Finland [31]. Corresponding results revealed the breast tumours that linked to $\mathrm{BM}$ do frequently express cytokeratin-5 $(P<0.05)$, prominin-1 $(P<0.05)$, nestin $(P<0.1)$, smooth muscle actin $(P<0.1)$ being more often ER and PR negative $(P<0.05)$. The heat shock protein $\alpha \mathrm{B}-$ crystallin (CRYAB) is frequently expressed in TN subtype of breast tumours being associated with early occurrence of BM and poor prognosis for the affected patients [32]. In an independent study utilising the data of $855 \mathrm{BC}$ patients, the CRYAB expression detected in the tumour tissue was proposed to be an independent predictor for BM as the first distant metastatic site (HR $=1.2,95 \%$ CI $1.0-1.4, P=0.021$ ) [33]. In a multivariable analysis including only patients with metastatic $\mathrm{BC}, \alpha \mathrm{B}$-crystallin was the strongest independent predictor of $\mathrm{BM}$ development $(\mathrm{HR}=2.99,95 \%$ CI 1.83 $4.89, P<0.0001)$.

Molecular biomarker panels involved in key steps of metastatic cascade are currently under extensive investigation: they regulate cancer cell motility, dissemination into the circulation, blood-brain barrier (BBB) disruption and brain seeding. An activation of potentially relevant signalling pathways, namely the phosphatidylinositol 3-kinase (PI3K) and mitogen-activated protein kinase (MAPK/ERK) ones, has been examined in BM cancer cells [34, 35]. Further, the overexpression of HER3 receptor and subsequent activation of MAPK signalling pathway were found to be preferentially activated in the brain metastases of $\mathrm{BC}$ patients [36, 37]. Preclinical studies demonstrated the stem cell-specific signalling by the Wnt and Notch pathways to be associated with the development of BM [38, 39]. Angiogenic factors- the vascular endothelial growth factor (VEGF), angiopoietin-2 and chemokine receptor type 4 (CXCR-4) - have been identified as the specific drivers of BM developmental process [40-45]. Dysregulation of these factors helps cancer cells to disrupt the blood-brain barrier followed by their extravasation and migration into the brain parenchyma.

A specific cellular and molecular repertoire within the organ characteristic local microenvironment predisposes the affected individuals to the appearance of the so-called pre-metastatic niches that might be the crucial step in the overall BM development [46]. This "fertile" microenvironment actively supporting the cancer advancement is strongly promoted by systemic hypoxic effects frequently appearing in individuals with cardiovascular disease and related syndromes being a powerful stratification factor for the predisposed individuals as recently reviewed by $\mathrm{O}$. Golubnitschaja et al. [1].

Further, a series of studies demonstrated that BC cells that invaded and seeded into the brain tissue are capable to "mimic" the phenotype of the neuronal cells by acquiring the ability to utilise the specific neurotransmitter which is the $\gamma$-aminobutyric acid (GABA) [47, 48]. Consequently, the invading breast cancer cells exhibit specific neural characteristics which enable them to penetrate into the brain tissue; thereby, highly activated GABA expression increases their proliferative capacity, particularly within the pre-metastatic niches as the preferable microenvironment. In this context, an active communication between invading cancer cells, astrocytes and microglia in the "hosting" brain parenchyma has been identified representing an essential part of the successful cancer cell nesting and metastatic process [49-52].

Gene expression and subsequent functional analyses of BC cells preferentially infiltrating the brain of the patients with advanced disease led to the identification of the cyclooxygenase-2 (COX2), the epidermal growth factor receptor (EGFR) ligand HBEGF and the $\alpha 2,6$-sialyltransferase (ST6GALNAC5) to be the specific genetic drivers of BM development [53]. The expression of these genes was functionally associated with the $\mathrm{BC}$ cell extravasation through the bloodbrain barrier breakdown. Although HBEGF and COX2 expression have been earlier identified as being linked to $\mathrm{BC}$ lung metastases, ST6GALNAC5 is proposed to play the role of the brain-specific mediator enabling the invasion of the cancer cells 
into this organ [53, 54]. Another study revealed organ-specific molecular signatures of $\mathrm{BC}$ cells which might be predictive for invading the brain, lung and liver [55]. At the cellular level, a development of the brain and lung metastases was associated with less differentiated but more stem cell-like characteristics of $\mathrm{BC}$ cells.

Recently performed whole exome sequencing of 86 matched $\mathrm{BM}$, primary BC tumours and normal tissue samples [56] demonstrated the branched evolution of tumour genetic alterations, since the metastases shared a common ancestor being, however, subsequently evolved compared to the original profiles of the primary $\mathrm{BC}$ tumours. To this end, the genetic alterations additionally developed in metastatic cells against the primary $\mathrm{BC}$ tumour cells were found in as many as $53 \%$ of cases investigated in the study. Moreover, the genetic profiles of extracranial and lymph node metastases were highly divergent from those identified in BM. An independent study analysing genomic profiling, gene expression and DNA methylation of $\mathrm{BM}$ revealed highly specific BM molecular profiles in BC patients [57]. Therefore, individualised molecular biological profiling of $\mathrm{BM}$ is clinically relevant for targeted therapy approaches and treatment algorithms tailored to the person.

\section{The targeted therapy for HER2-positive breast cancer in view of the brain metastasis risk}

Paradoxically, the targeted anti-HER2 therapy applied to the HER2-positive BC patients may be associated with an increased risk of BM development: four studies dedicated to the meta-analyses of large adjuvant trastuzumab trials found 1.3 - to 1.8-fold increased risk of BM as the first metastatic site [58-61]. On the other side, the retrospective analysis showed no significant difference in BM development as the first site of recurrence amongst HER2-positive BC patients receiving 1-yearlong adjuvant trastuzumab against placebo ( 2 vs. $2 \%$, respectively, $P=0.55)$ [62]. Moreover, adjuvant trastuzumab significantly reduces the non-brain recurrences $(P<0.0001)$. Further, the CLEOPATRA study explored the efficacy of the dual antiHER2 inhibition combining trastuzumab and pertuzumab application compared to treatment by trastuzumab alone- both in combination with the docetaxel applied to the first-line metastatic HER2-positive BC patients [63]. Although the results demonstrated the similar incidence of BM (12.6 placebo arm vs. $13.7 \%$ pertuzumab arm), the time to the BM development was prolonged in the pertuzumab arm from 11.9 to 15 months (HR $=0.58,95 \%$ CI $0.39-0.85, P=0.0049)$ [64]. The CEREBEL clinical trial compared an efficacy of the HER2 tyrosine kinase inhibitor lapatinib application against trastuzumab one, both in combination with capecitabine, in metastatic BC patients initially without detectable BM [65]. The study was designed investigating the short-term effects. The subsequent results have demonstrated no significant difference between two arms regarding the BM development (3\% lapatinib arm vs. $5 \%$ trastuzumab arm, $P=0.36$ ). Although the question is not definitively answered, it has been concluded that an increased incidence of BM in BC patients that underwent the targeted anti-HER2 therapeutics seems to result from the prolonged survival of the treated patients rather than being a negative side effect of the drugs applied.

\section{Conclusions, outlook and expert recommendations}

It becomes increasingly apparent that the progression of initially localised $\mathrm{BC}$ into the central nervous system is a very complex process. The whole metastatic cascade involves primary cancer cell dissemination into the circulation, attachment to brain capillaries, the blood-brain barrier disruption, extravasation and, finally, brain seeding and proliferation into the new metastatic lesions $[28,66]$. During this process, cancer cells undergo a number of modifications at both genetic and epigenetic levels.

Further, the local microenvironment of the brain parenchyma plays a central role in the development of BM. In particular, systemic hypoxia strongly contributes to the "fertile microenvironment" creating pre-metastatic niches in distant organs $[46,67]$. Therefore, it has been highly recommended to select and stratify potentially predisposed individuals who may suffer from the systemic hypoxic effects such as CVD patients and "Flammer syndrome" individuals, in order to predict and prevent particularly aggressive cancer types and fatal metastatic disease [68]. To this end, it is notable that the highest frequency of BM amongst metastatic BC patients is recorded to be by $20-30 \%$ in the triple-negative breast cancer patients [21-23], who are currently under extensive investigation regarding their strong phenotype-dependent predisposition to the system hypoxic effects and, therefore, to the molecular mechanisms "fertilising" the local microenvironment for a particularly aggressive cancer advancement [68].

Future developments in the area certainly will be based on the following:

- The multi-professional collaboration

- Application of individualised patient profiles

- Improved patient stratification

- Predictive approaches

- Targeted prevention at the level of initial tumours in breast as well as timely measures tailored to the person, in order to protect the predisposed breast cancer patients against the attack by the circulating tumour cell spread and formation of the fertile pre-metastatic niches in distant organs such as the brain.

In this context, innovative multilevel diagnostic approaches are needed to predict $\mathrm{BC}$ patients at high risk for potential BM development. To this end, summarised 


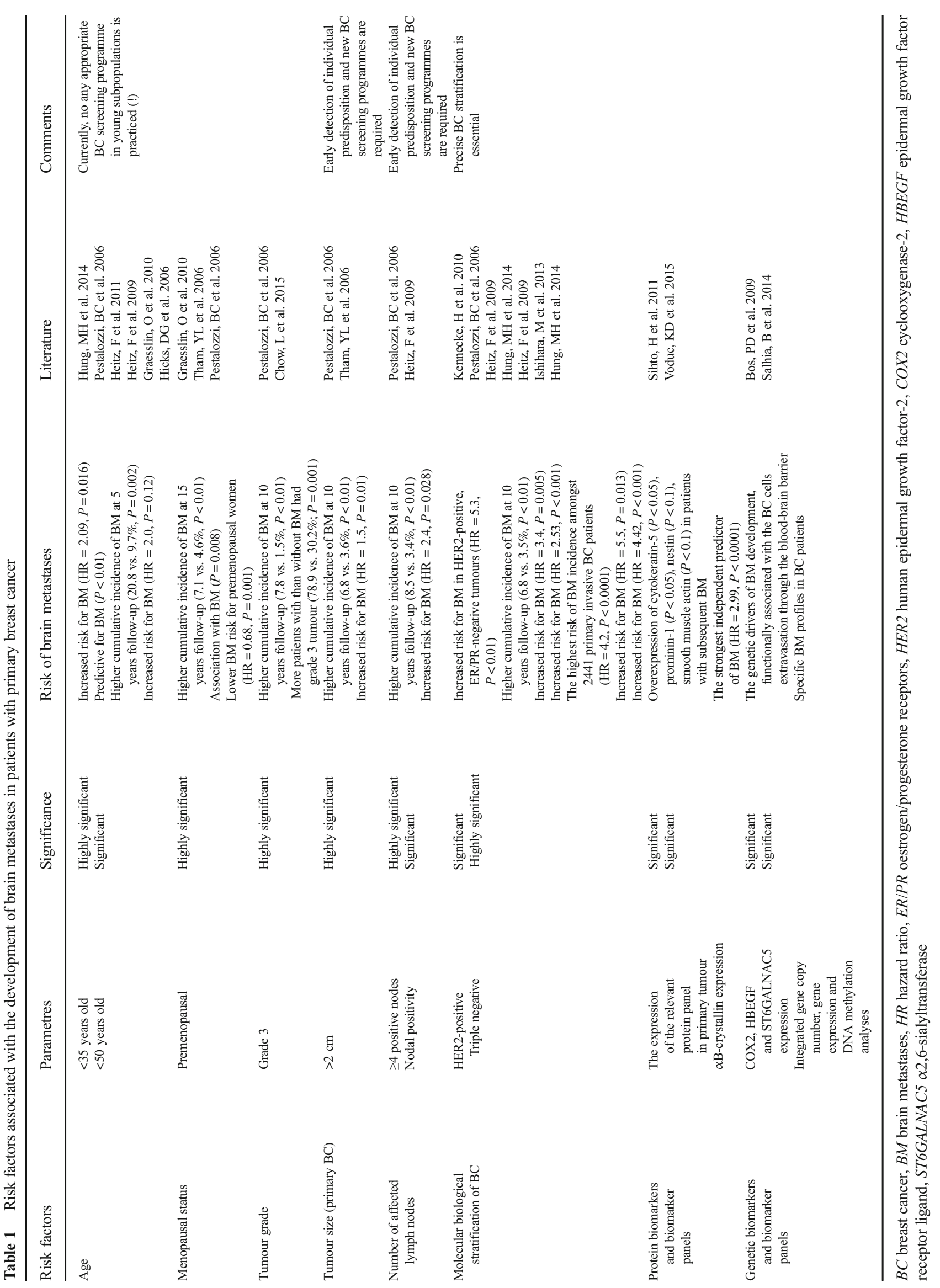


information is presented in Table 1. Multi-parametric models assessing BM risks are currently under consideration [12]. Further, the multi-parametric prognostic tools for the optimised BM treatment algorithms, disease and therapy monitoring are under development [69-71]. The above proposed measures may substantially increase the quality of life of the predisposed individuals and affected patients by increasing the efficacy and reducing the overall costs of the healthcare in the area.

Acknowledgements This work was supported by the MH CZ-DRO (Faculty Hospital in Plzen-FNPl, 00669806) and by the National Sustainability Program I (NPU I) Nr. LO1503 provided by the Ministry of Education Youth and Sports of the Czech Republic.

Authors' contributions OG is the project coordinator who has created the main scientific ideas and hypotheses presented in the manuscript. JP Jr. has performed the literature search, analysed the data and drafted the manuscript. OG and JP Jr. have designed the final version of the manuscript. CK and WK have contributed to the manuscript by their expertise in breast cancer research and treatment. MK and JP have provided expertise to the multilevel diagnostics. All the authors have read and approved the final manuscript

\section{Compliance with ethical standards}

Conflict of interest The authors declare that they have no conflict of interest.

Statement of informed consent Patients have not been involved in the study.

Statement of human and animal rights No experiments have been performed including patients and/or animals.

\section{References}

1. Golubnitschaja O, Debald M, Yeghiazaryan K, Kuhn W, Pešta M, Costigliola V, et al. Breast cancer epidemic in the early twenty-first century: evaluation of risk factors, cumulative questionnaires and recommendations for preventive measures. Tumour Biol J Int Soc Oncodevelopmental Biol Med. 2016;37:12941-57.

2. Darlix A, Zouaoui S, Rigau V, Bessaoud F, Figarella-Branger D, Mathieu-Daudé $\mathrm{H}$, et al. Epidemiology for primary brain tumors: a nationwide population-based study. J Neurooncol. 2016.

3. Polivka J, Polivka J, Rohan V, Topolcan O. New treatment paradigm for patients with anaplastic oligodendroglial tumors. Anticancer Res. 2014;34:1587-94.

4. Polivka J, Polivka Jr J, Rohan V, Topolcan O. Glioblastoma multiforme - a review of pathogenesis, biomarkers and therapeutic perspectives. Cesk Slov Neurol N. 2013;76(109):575-83.

5. Polivka J, Polivka J, Holubec L, Kubikova T, Priban V, Hes O, et al. Advances in experimental targeted therapy and immunotherapy for patients with glioblastoma multiforme. Anticancer Res. 2017;37:21-33.

6. Lin NU, Bellon JR, Winer EP. CNS metastases in breast cancer. J Clin Oncol Off J Am Soc Clin Oncol. 2004;22:3608-17.

7. Chang EL, Lo S. Diagnosis and management of central nervous system metastases from breast cancer. Oncologist. 2003;8:398-410
8. Hung M-H, Liu C-Y, Shiau C-Y, Hsu C-Y, Tsai Y-F, Wang Y-L, et al. Effect of age and biological subtype on the risk and timing of brain metastasis in breast cancer patients. PLoS One. 2014;9:e89389.

9. Pestalozzi BC, Zahrieh D, Price KN, Holmberg SB, Lindtner J, Collins $\mathrm{J}$, et al. Identifying breast cancer patients at risk for Central Nervous System (CNS) metastases in trials of the International Breast Cancer Study Group (IBCSG). Ann Oncol Off J Eur Soc Med Oncol. 2006;17:935-44.

10. Tham Y-L, Sexton K, Kramer R, Hilsenbeck S, Elledge R. Primary breast cancer phenotypes associated with propensity for central nervous system metastases. Cancer. 2006;107:696-704.

11. Heitz F, Rochon J, Harter P, Lueck H-J, Fisseler-Eckhoff A, Barinoff J, et al. Cerebral metastases in metastatic breast cancer: disease-specific risk factors and survival. Ann Oncol Off J Eur Soc Med Oncol. 2011;22:1571-81.

12. Graesslin O, Abdulkarim BS, Coutant C, Huguet F, Gabos Z, Hsu $\mathrm{L}$, et al. Nomogram to predict subsequent brain metastasis in patients with metastatic breast cancer. J Clin Oncol Off J Am Soc Clin Oncol. 2010;28:2032-7.

13. Hicks DG, Short SM, Prescott NL, Tarr SM, Coleman KA, Yoder BJ, et al. Breast cancers with brain metastases are more likely to be estrogen receptor negative, express the basal cytokeratin CK $5 / 6$, and overexpress HER2 or EGFR. Am J Surg Pathol. 2006;30:1097-104.

14. Sperduto PW, Kased N, Roberge D, Xu Z, Shanley R, Luo X, et al. Summary report on the graded prognostic assessment: an accurate and facile diagnosis-specific tool to estimate survival for patients with brain metastases. J Clin Oncol Off J Am Soc Clin Oncol. 2012;30:419-25.

15. Chow L, Suen D, Ma KK, Kwong A. Identifying risk factors for brain metastasis in breast cancer patients: implication for a vigorous surveillance program. Asian J Surg. 2015;38:220-3.

16. Heitz F1, Harter P, Lueck HJ, Fissler-Eckhoff A, Lorenz-Salehi F, Scheil-Bertram S, et al. Triple-negative and HER2-overexpressing breast cancers exhibit an elevated risk and an earlier occurrence of cerebral metastases. Eur J Cancer. 2009;45(16):2792-8. doi:10. 1016/j.ejca.2009.06.027.

17. Prevention of breast cancers summary | European Cancer Prevention [Internet]. [cited 2016 Dec 30]. Available from: http:// ecpo.org/?page id $=522$

18. Perou CM, Sørlie T, Eisen MB, van de Rijn M, Jeffrey SS, Rees CA, et al. Molecular portraits of human breast tumours. Nature. 2000;406:747-52.

19. Network CGA. Comprehensive molecular portraits of human breast tumours. Nature. 2012;490:61-70.

20. Makki J. Diversity of breast carcinoma: histological subtypes and clinical relevance. Clin Med Insights Pathol. 2015;8:23-31.

21. Kennecke H, Yerushalmi R, Woods R, Cheang MCU, Voduc D, Speers $\mathrm{CH}$, et al. Metastatic behavior of breast cancer subtypes. J Clin Oncol Off J Am Soc Clin Oncol. 2010;28:3271-7.

22. Smid M, Wang Y, Zhang Y, Sieuwerts AM, Yu J, Klijn JGM, et al. Subtypes of breast cancer show preferential site of relapse. Cancer Res. 2008;68:3108-14.

23. Soni A, Ren Z, Hameed O, Chanda D, Morgan CJ, Siegal GP, et al. Breast cancer subtypes predispose the site of distant metastases. Am J Clin Pathol. 2015;143:471-8.

24. Ishihara M, Mukai H, Nagai S, Onozawa M, Nihei K, Shimada T, et al. Retrospective analysis of risk factors for central nervous system metastases in operable breast cancer: effects of biologic subtype and Ki67 overexpression on survival. Oncology. 2013;84:135-40.

25. Tonyali O, Coskun U, Yuksel S, Inanc M, Bal O, Akman T, et al. Risk factors for brain metastasis as a first site of disease recurrence in patients with HER 2 positive early stage breast cancer treated with adjuvant trastuzumab. Breast Edinb Scotl. 2016;25:22-6.

26. Leone JP, Leone BA. Breast cancer brain metastases: the last frontier. Exp Hematol Oncol. 2015;4:33. 
27. Venur VA, Leone JP. Targeted therapies for brain metastases from breast cancer. Int J Mol Sci. 2016;17.

28. Witzel I, Oliveira-Ferrer L, Pantel K, Müller V, Wikman H. Breast cancer brain metastases: biology and new clinical perspectives. Breast Cancer Res BCR. 2016;18:8.

29. Polivka J, Pesta M, Janku F. Testing for oncogenic molecular aberrations in cell-free DNA-based liquid biopsies in the clinic: are we there yet? Expert Rev Mol Diagn. 2015;15:1631-44.

30. Sosińska-Mielcarek K, Duchnowska R, Winczura P, Badzio A, Majewska H, Lakomy J, et al. Immunohistochemical prediction of brain metastases in patients with advanced breast cancer: the role of Rad51. Breast Edinb Scotl. 2013;22:1178-83.

31. Sihto H, Lundin J, Lundin M, Lehtimäki T, Ristimäki A, Holli K, et al. Breast cancer biological subtypes and protein expression predict for the preferential distant metastasis sites: a nationwide cohort study. Breast Cancer Res BCR. 2011;13:R87.

32. Malin D, Strekalova E, Petrovic V, Deal AM, Al Ahmad A, Adamo B, etal. $\alpha \mathrm{B}$-crystallin: a novel regulator of breast cancer metastasis to the brain. Clin Cancer Res Off J Am Assoc Cancer Res. 2014;20:56-67.

33. Voduc KD, Nielsen TO, Perou CM, Harrell JC, Fan C, Kennecke H, et al. $\alpha \mathrm{B}$-crystallin expression in breast cancer is associated with brain metastasis. NPJ Breast Cancer. 2015;1.

34. Adamo B, Deal AM, Burrows E, Geradts J, Hamilton E, Blackwell KL, et al. Phosphatidylinositol 3-kinase pathway activation in breast cancer brain metastases. Breast Cancer Res BCR. 2011;13:R125.

35. Sirkisoon SR, Carpenter RL, Rimkus T, Miller L, Metheny-Barlow L, Lo H-W. EGFR and HER2 signaling in breast cancer brain metastasis. Front Biosci Elite Ed. 2016;8:245-63.

36. Da Silva L, Simpson PT, Smart CE, Cocciardi S, Waddell N, Lane A, et al. HER3 and downstream pathways are involved in colonization of brain metastases from breast cancer. Breast Cancer Res BCR. 2010;12:R46.

37. Berghoff AS, Bartsch R, Preusser M, Ricken G, Steger GG, BagoHorvath Z, et al. Co-overexpression of HER2/HER3 is a predictor of impaired survival in breast cancer patients. Breast Edinb Scotl. 2014;23:637-43.

38. Nam D-H, Jeon H-M, Kim S, Kim MH, Lee Y-J, Lee MS, et al. Activation of notch signaling in a xenograft model of brain metastasis. Clin Cancer Res Off J Am Assoc Cancer Res. 2008;14:4059-66.

39. McGowan PM, Simedrea C, Ribot EJ, Foster PJ, Palmieri D, Steeg PS, et al. Notch1 inhibition alters the CD44hi/CD24lo population and reduces the formation of brain metastases from breast cancer. Mol Cancer Res MCR. 2011;9:834-44.

40. Lee T-H, Avraham HK, Jiang S, Avraham S. Vascular endothelial growth factor modulates the transendothelial migration of MDAMB-231 breast cancer cells through regulation of brain microvascular endothelial cell permeability. J Biol Chem. 2003;278:5277-84.

41. Avraham HK, Jiang S, Fu Y, Nakshatri H, Ovadia H, Avraham S. Angiopoietin-2 mediates blood-brain barrier impairment and colonization of triple-negative breast cancer cells in brain. J Pathol. 2014;232:369-81.

42. Lee B-C, Lee T-H, Avraham S, Avraham HK. Involvement of the chemokine receptor CXCR4 and its ligand stromal cell-derived factor 1alpha in breast cancer cell migration through human brain microvascular endothelial cells. Mol Cancer Res MCR. 2004;2:327-38.

43. Xu C, Zhao H, Chen H, Yao Q. CXCR4 in breast cancer: oncogenic role and therapeutic targeting. Drug Des Devel Ther. 2015;9:4953-64.

44. Wrobel JK, Toborek M. Blood-brain barrier remodeling during brain metastasis formation. Mol Med Camb Mass. 2016.

45. Kim LS, Huang S, Lu W, Lev DC, Price JE. Vascular endothelial growth factor expression promotes the growth of breast cancer brain metastases in nude mice. Clin Exp Metastasis. 2004;21:107-18.

46. Cox TR, Rumney RMH, SchoofEM, Perryman L, Høye AM, Agrawal A, et al. The hypoxic cancer secretome induces pre-metastatic bone lesions through lysyl oxidase. Nature. 2015;522:106-10.
47. Neman J, Termini J, Wilczynski S, Vaidehi N, Choy C, Kowolik $\mathrm{CM}$, et al. Human breast cancer metastases to the brain display GABAergic properties in the neural niche. Proc Natl Acad Sci U S A. 2014;111:984-9.

48. Van Swearingen AE, Siegel MB, Anders CK. Breast cancer brain metastases: evidence for neuronal-like adaptation in a "breast-tobrain" transition? Breast Cancer Res BCR. 2014;16:304.

49. Termini J, Neman J, Jandial R. Role of the neural niche in brain metastatic cancer. Cancer Res. 2014;74:4011-5.

50. Pukrop T, Dehghani F, Chuang H-N, Lohaus R, Bayanga K, Heermann $\mathrm{S}$, et al. Microglia promote colonization of brain tissue by breast cancer cells in a Wnt-dependent way. Glia. 2010;58:1477-89.

51. Fitzgerald DP, Palmieri D, Hua E, Hargrave E, Herring JM, Qian Y, et al. Reactive glia are recruited by highly proliferative brain metastases of breast cancer and promote tumor cell colonization. Clin Exp Metastasis. 2008;25:799-810.

52. Neman J, Choy C, Kowolik CM, Anderson A, Duenas VJ, Waliany $\mathrm{S}$, et al. Co-evolution of breast-to-brain metastasis and neural progenitor cells. Clin Exp Metastasis. 2013;30:753-68.

53. Bos PD, Zhang XH-F, Nadal C, Shu W, Gomis RR, Nguyen DX, et al. Genes that mediate breast cancer metastasis to the brain. Nature. 2009;459:1005-9.

54. Minn AJ, Gupta GP, Siegel PM, Bos PD, Shu W, Giri DD, et al. Genes that mediate breast cancer metastasis to lung. Nature. 2005;436:518-24.

55. Harrell JC, Prat A, Parker JS, Fan C, He X, Carey L, et al. Genomic analysis identifies unique signatures predictive of brain, lung, and liver relapse. Breast Cancer Res Treat. 2012;132:523-35.

56. Brastianos PK, Carter SL, Santagata S, Cahill DP, Taylor-Weiner A, Jones RT, et al. Genomic characterization of brain metastases reveals branched evolution and potential therapeutic targets. Cancer Discov. 2015;5:1164-77.

57. Salhia B, Kiefer J, Ross JTD, Metapally R, Martinez RA, Johnson $\mathrm{KN}$, et al. Integrated genomic and epigenomic analysis of breast cancer brain metastasis. PLoS One. 2014;9, e85448.

58. Olson EM, Abdel-Rasoul M, Maly J, Wu CS, Lin NU, Shapiro CL. Incidence and risk of central nervous system metastases as site of first recurrence in patients with HER2-positive breast cancer treated with adjuvant trastuzumab. Ann Oncol Off J Eur Soc Med Oncol. 2013;24:1526-33.

59. Bria E, Cuppone F, Fornier M, Nisticò C, Carlini P, Milella M, et al. Cardiotoxicity and incidence of brain metastases after adjuvant trastuzumab for early breast cancer: the dark side of the moon? A meta-analysis of the randomized trials. Breast Cancer Res Treat. 2008;109:231-9.

60. Viani GA, Afonso SL, Stefano EJ, De Fendi LI, Soares FV. Adjuvant trastuzumab in the treatment of her-2-positive early breast cancer: a meta-analysis of published randomized trials. BMC Cancer. 2007;7:153.

61. Dahabreh IJ, Linardou H, Siannis F, Fountzilas G, Murray S. Trastuzumab in the adjuvant treatment of early-stage breast cancer: a systematic review and meta-analysis of randomized controlled trials. Oncologist. 2008;13:620-30.

62. Pestalozzi BC, Holmes E, de Azambuja E, Metzger-Filho O, Hogge $\mathrm{L}$, Scullion M, et al. CNS relapses in patients with HER2-positive early breast cancer who have and have not received adjuvant trastuzumab: a retrospective substudy of the HERA trial (BIG 101). Lancet Oncol. 2013;14:244-8.

63. Swain SM, Kim S-B, Cortés J, Ro J, Semiglazov V, Campone M, et al. Pertuzumab, trastuzumab, and docetaxel for HER2-positive metastatic breast cancer (CLEOPATRA study): overall survival results from a randomised, double-blind, placebo-controlled, phase 3 study. Lancet Oncol. 2013;14:461-71.

64. Swain SM, Baselga J, Miles D, Im Y-H, Quah C, Lee LF, et al. Incidence of central nervous system metastases in patients with HER2-positive metastatic breast cancer treated with pertuzumab, 
trastuzumab, and docetaxel: results from the randomized phase III study CLEOPATRA. Ann Oncol Off J Eur Soc Med Oncol. 2014;25:1116-21.

65. Pivot X, Manikhas A, Żurawski B, Chmielowska E, Karaszewska B, Allerton R, et al. CEREBEL (EGF111438): a phase III, randomized, open-label study of lapatinib plus capecitabine versus trastuzumab plus capecitabine in patients with human epidermal growth factor receptor 2-positive metastatic breast cancer. J Clin Oncol Off J Am Soc Clin Oncol. 2015;33:1564-73.

66. Tayyeb B, Parvin M. Pathogenesis of breast cancer metastasis to brain: a comprehensive approach to the signaling network. Mol Neurobiol. 2016;53:446-54.

67. Vanharanta S. A hypoxic ticket to the bone metastatic niche. Breast Cancer Res BCR. 2015;17:122.

68. Golubnitschaja O, Debald M, Kuhn W, Yeghiazaryan K, Bubnov RV, Goncharenko VM, et al. Flammer syndrome and potential formation of pre-metastatic niches: a multi-centred study on phenotyping, patient stratification, prediction and potential prevention of aggressive breast cancer and metastatic disease. EPMA J. 2016;7:A25.

69. Marko NF, Xu Z, Gao T, Kattan MW, Weil RJ. Predicting survival in women with breast cancer and brain metastasis: a nomogram outperforms current survival prediction models. Cancer. 2012;118:3749-57.

70. Subbiah IM, Lei X, Weinberg JS, Sulman EP, Chavez-MacGregor $\mathrm{M}$, Tripathy $\mathrm{D}$, et al. Validation and development of a modified breast graded prognostic assessment as a tool for survival in patients with breast cancer and brain metastases. J Clin Oncol Off J Am Soc Clin Oncol. 2015;33:2239-45.

71. Barnholtz-Sloan JS, Yu C, Sloan AE, Vengoechea J, Wang M, Dignam $\mathrm{JJ}$, et al. A nomogram for individualized estimation of survival among patients with brain metastasis. Neuro-Oncology. 2012;14:910-8. 